\title{
Transforming Service Delivery with TOGAF and Archimate in a Government Agency in Peru
}

\author{
Jorge Valenzuela Posadas \\ INTILab, Universidad de Ciencias y Humanidades - UCH, Lima- Peru
}

\begin{abstract}
The application of The Open Group Architecture Framework (TOGAF) and Archimate to transform the citizen's service delivery by the Ministry of Labor and Employment Promotion of Peru is presented. The enterprise architecture development has followed the phases of the TOGAF Architecture Development Method (ADM) method: Architecture Vision, Business, Data, Application and Technology Architecture Definition to determine the source architecture, the target architecture and gaps to meeting the target architecture requirements. The strategic motivations, active structure, passive structure, behavior and different viewpoints models in the domains: business, data, application and technology have been constructed with the Archimate descriptive language. The viewpoints achieved by the conjunction of these open standards have allowed identifying fragmented and isolated business services, as well as duplicated data and duplicating application functions. A proposal for integrated and transversal services emerges as a result of the applied Enterprise Architecture approach. Design science is applied to obtain knowledge from the generated artifacts. The knowledge generated from this application can be useful for new initiatives to improve the delivery of services to the citizen in the Peruvian government.
\end{abstract}

Keywords-Enterprise architecture; business architecture; digital government; The Open Group Architecture Framework (TOGAF); archimate; design science

\section{INTRODUCTION}

In 2016, the Organization for Economic and Co-operation and Development (OECD) elaborate the "OECD Public Governance Reviews: Peru-Integrated Governance for Inclusive Growth" [1] where it recommends that Peru should follow a comprehensive reform agenda of the public administration, which consists in taking advantage of digital government to achieve open and transparent government capacities, in order to strengthen the making decisions, reducing costs and improving the quality of citizens services.

Despite the advances, the diagnosis that the OECD delivers to Peru revolves around its current approach that limits the achievement of strategies and integration: "the consequence of adopting a procedural, legalistic and technical approach to digital technology may be undermining the achievement of more strategic and integrated results while the government carries out its modernization efforts in this area "[1].

An Enterprise Architecture (EA) provides an understandable representation of the structure and strategic motivation of a purposeful activity, both in an organization or in a transversal functional area [2]. An EA is an essential tool to effectively and efficiently design business and missional processes and to implement and evolve information systems. There are $67 \%$ of countries in the world in the process of developing their EA or similar program to improve interoperability around public administration information systems, mitigate duplicate information, and maximize return on investment [3].

In Peru, as part of responding to the recommendations of the OECD regarding improving its digital government capabilities, the Digital Government Law has been enacted [3], whose main objective is achieve cross-cutting citizen's services with the intensive use of digital technology in government agencies.

Many countries encourage their government agencies and local governments to introduce enterprise architecture approach, developing principles, standards, frameworks, and reference models. Meanwhile, other more advanced countries have made the effort to develop a government-wide enterprise architecture [4] which is encouraged to provide a comprehensive view of enterprise architecture to their agencies [5]. This is the case of: Federal Enterprise Architecture (FEAF) [6], Australian Government Architecture (AGA) [7], United Kingdom cross-Government Enterprise Architecture (xGEA) [8], Singapore Government Enterprise Architecture (SGEA) [9], Korea Government-wide Enterprise Architecture (GEA) [4]. India Enterprise Architecture (IndEA) [10], Business Architecture Reference Framework (AE) for IT Management in Colombia [11] and others. In Peru, a government-wide architecture framework has not yet been stablished, and there is still no clear guidance on how achieve it, which is difficult due to the fact that there is not a CIO body of government with competences in Enterprise Architecture.

In the world, there are initiatives to apply enterprise architecture at a sector level, that is, in a government agency and in local governments; e.g. U.S. Office of Personal Management with the establishment of the Human Resources Line of Business (HR LOB) [12], HR LOB has used enterprise architecture to drive the transformation towards a new Human Resources service delivery model by crossing the US federal government in 2004.

The current initiative of the Ministry of Labor and Employment Promotion of Peru, regarding the development of its enterprise architecture, is motivated by: 1) Reduce the complexity of information systems by ensuring alignment with strategic institutional motivations, redesign and delivery of transversal services to the citizen, uniformed applications and standardized data (without duplication), virtualization consolidated and distributed, technological support for 
interoperability, scalability and take advantage of the elasticity of the Cloud. 2) Reduce information systems maintenance costs and; 3) Manage efficiently institutional and technological change.

The research methodology used in this work is Design Science, as proposed in [13]. The next section presents the related work on applying TOGAF and ARCHIMATE to transform the delivery of services in the public sector. Design science is applied, to obtain knowledge of the constructed artifacts of the definition of enterprise architecture in the Peruvian labor agency, with its corresponding demonstration and evaluation. The knowledge generated in this work can serve as lessons learned to support new and next similar initiatives.

\section{RELATED WORK}

The implementation of enterprise architecture in the health sector of Norway, indicates that when the roles of enterprise architecture are not established, there is not effective communication, there is weak commitment and maturity with EA and there is difficult to use EA tools, all this can impede the process towards the achievement and adoption of an EA common [14].

Government agencies typically have different structures and business areas / services that often lead to duplicate information systems and fragmented business processes and services, reducing the possibility of cross-agency interoperability [14]. Therefore, governments pursue EA initiatives, in addition to the aforementioned general EA benefits, to enable their business processes to be carried out from start to finish, across all government agencies, to increase online services [15] to provide new tools to manage business; and IT alignment with agencies for better integration of technologies, to rationalize data and application structure and to provide business modularity[16].

Another application of EA in the public sectors of labor and welfare, hospital and health of Norway concludes that autonomy, financial matters and the understanding of EA, are the three most important aspects to address success with EA in the public sector [17].

There are few examples of practical use with architecture development frameworks, modeling of designed artifacts and the reasonableness to identify architectural gaps [18]. Few works present the rationality of a regular application of enterprise architecture to a government agency to verify the benefits of simplification and change management that theoretically offers.

As mentioned in the introduction, the research methodology used in this work is Design Science Research Method (DSRM) where a research proposal is developed and validated to solve a problem [13]. This methodology is an iterative research process (as Table I shows) with the goal of overcoming standard research methodologies, such as traditional descriptive research and interpretive research, whose research outputs are largely explanatory and often not applicable to problem solving in practice [18]. DSRM covers this gap with design and creation of an applicable solution explicit to a problem, being an accepted research paradigm in the engineering area.
TABLE I. DESIGN SCIENCE RESEARCH METHODOLOGY

\begin{tabular}{|l|l|}
\hline Phase & Description \\
\hline $\begin{array}{l}\text { Define the problem } \\
\text { and motivation }\end{array}$ & Define problem and show the importance \\
\hline $\begin{array}{l}\text { Define objectives of a } \\
\text { solution }\end{array}$ & What would a better artifact to accomplish? \\
\hline $\begin{array}{l}\text { Design and } \\
\text { development }\end{array}$ & Artifact \\
\hline Demonstration & Find suitable context, use artifact to solve problem \\
\hline Evaluation & $\begin{array}{l}\text { Observe how effective and efficient, Iterate back to } \\
\text { design }\end{array}$ \\
\hline Communication & Scholarly publications, professional publications \\
\hline
\end{tabular}

Archimate delivers a uniform representation of EA models and views, offers an integrated architecture approach that describes and visualizes all architectural domains, their relationships and dependencies delivering different viewpoints for selected stakeholders, as well as support being extended.

\section{PROBlem DEFINITION}

Currently, Ministry of Labor and Employment Promotion of Peru maintains 105 applications and a data center with 37 physical servers and 66 virtual servers. The agency has two business divisions: regulatory labor and employment promotion. In front of the need for information, the entity generates mechanisms and rules to collect information from working citizens. This can be through a service (worker formalization, labor intermediation, employer registration, job offer registration, etc.) or through a regulatory obligation (registration of a civil construction worker, etc.). Due to a defragmentation of efforts, news applications are developed for the news initiatives of each business division. As a result, there is a duplication of information, repetition of the same functionality in different applications, complex and disaggregated services to the citizen.

There is no shared representation of this reality, which allows identifying the problematic situation in a standard language for all stakeholders and with well-defined concepts to achieve common understanding of the problem and proposed solutions. There is no architectural development method that enables capacities for the diagnosis of gaps, establishment of a desired architecture, refinement of solutions and opportunities, a migration plan and the governance of the same in the aforementioned government entity.

\section{PRoposed Solution}

The objective of the proposed solution under the enterprise architecture approach in the aforementioned government entity is: 1) Ensure alignment between strategic motivators and business processes and services. 2) Ensure alignment between citizen's services and business processes with applications, data and technological infrastructure underlying. 3) Achieve and contribute to redesign the citizen's cross-cutting services and simplify, standardize and interoperate the corresponding data, applications and technological infrastructure. 


\section{TOGAF, ARCHIMATE AND VIEWPOINTS}

To achieve the objective of the proposed solution, it was required to carry out the phases of the TOGAF ADM, the initial evaluation of architectural capabilities, the architecture vision and principles, the business, data, application and infrastructure architecture definition. This phase allows identify opportunities and candidate solutions as effective responses to architecture requirements and gaps. At present work, the resulting viewpoints modeled with Archimate are presented with its rationality, to justify the paper proposed solutions.

The most significant viewpoints are presented with a topdown approach from the strategic motivations towards the architecture of information systems.

\section{A. Motivational Viewpoint}

The stakeholders that correspond to the two business divisions are identified: Labor regulation and employment promotion, whose motivators are the formalization of employment as well as strengthening the accessibility to work certificate. The goals to achieve are to increase the formal employment rate and the opportunities for the insertion of young workers. The requirements to achieve this are the workers' register and the unique work certificate for young workers. The principle of enterprise architecture is to maximize the benefit of the government agency as a whole, limited by the budget for results. Fig. 1 displays the motivational reality modelling as has been described.

\section{B. Strategies Viewpoint}

The courses of action are to promote the formalization of work as well as the employability of young workers through technical assistance and official certification of competencies, with the use of normative, supervisory and national employment centers. Showing the resources allocated to provide said capabilities. Fig. 2 presents the strategic reality modelling saving to match.

\section{Business Process Cooperation Viewpoint- AS IS}

The civil construction worker registration business service is presented in Fig. 3. Although pursue different strategies and satisfy the interests of different stakeholders, both services realizing common activities such as register a worker, and after a sequence of information validations. They deliver a printed certificate, upon completing the process flow.

At this point, two citizen's services (the second service is not presented due to space), which follow separate activities flows have been identified. In order to define the desired business architecture, it is necessary to make use of the architectural principles, which indicate "Establish crosssectional citizen's services integrating organizational units and business processes". Under that reasonableness the desired business architecture is designed, with a single worker registration process, and as part of the flow there is a procedure to typify the worker and capture the corresponding information, the flow ends with the issuance of a standardized record and with $100 \%$ digital technologies.

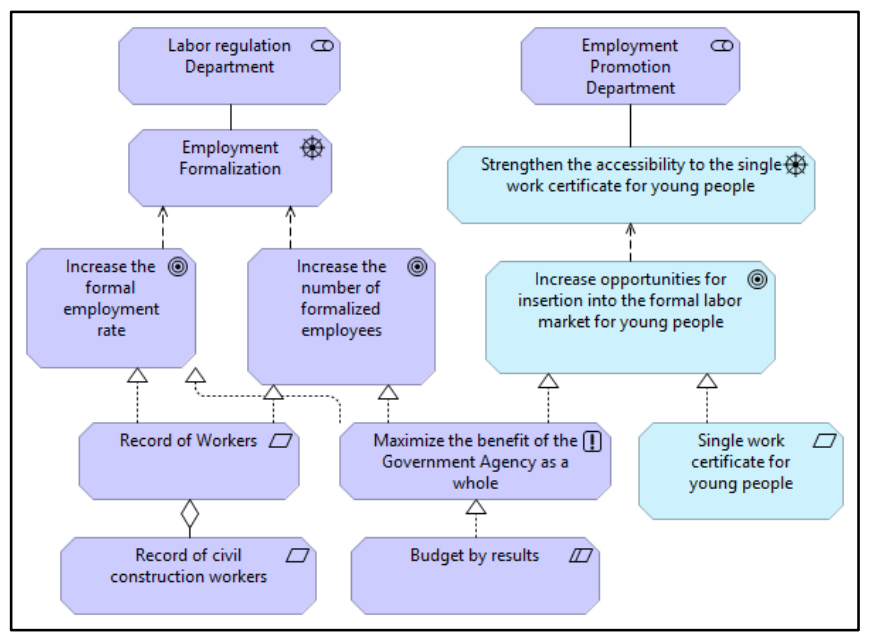

Fig. 1. Archimate Motivational Viewpoint.

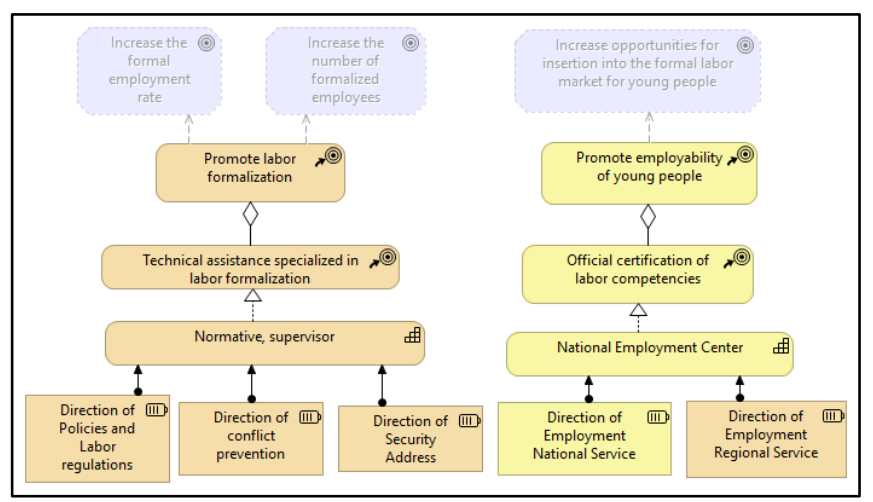

Fig. 2. Archimate Strategy Viewpoint.

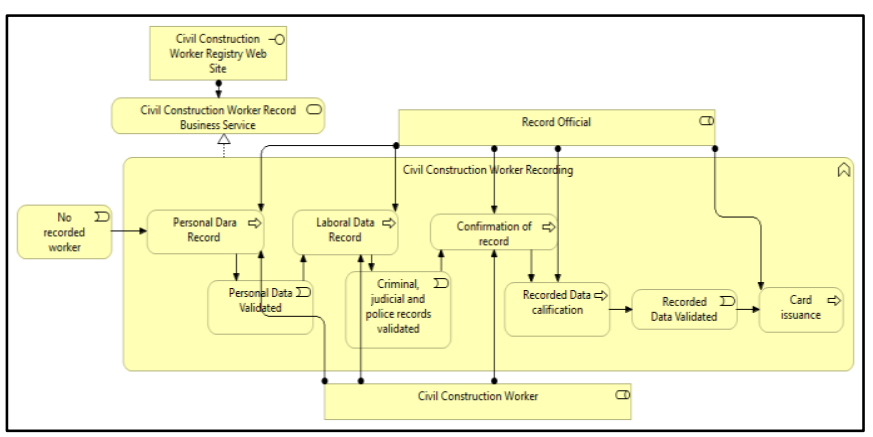

Fig. 3. Archimate Civil Construction Worker Recording Business Process Cooperation Viewpoint.

\section{Business Process Cooperation Viewpoint - TO BE}

Consider the standardization of the delivery of the transversal citizen's service. That is, the simplification of worker registration services. The new redesigned citizen's service is presented in Fig. 4. The process of worker registration is only one, and there is a new registration typing process where will get customizing information about worker such as required to business rules. The information validation process is centralized to abstract this rules for doing easy the reutilization and maintainability. 


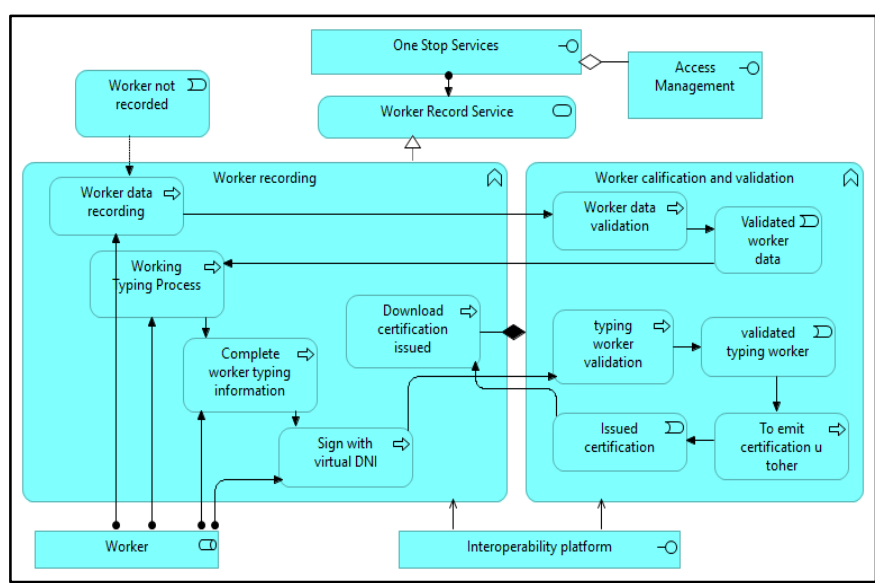

Fig. 4. Archimate TO BE Worker Recording Business Process Cooperation Viewpoint.

\section{E. Application Cooperation Viewpoint - AS IS}

It is appreciated that the current applications that support are duplicated at the level of application functionalities and redundant information but overall no standardized software component and libraries, such as Fig. 5 shows.

\section{F. Application Cooperation Viewpoint - TO BE}

From the Archimate representation, common components and standards for simplify the applications and removed duplicated functionality and redundant information has been identified. This will satisfy requirements for standardization, interoperability, scalability, security, orientation to services as well as functional. It consists of identifying components of common use and reusable as well as an exploration of solutions in the industry. The decoupling of the components facilitates maintenance as well as the most effective team work. The TO $\mathrm{BE}$ application cooperation replaces all the worker registration systems of the aforementioned government agency: civil construction worker registration, foreign worker's registration, SME worker registration, etc. Offering a cross-sectional worker registration service that can cross all public agencies in the medium term. Fig. 6 shows TO BE application cooperation viewpoint on Archimate language.

Table II shows the gap analysis developed after the realization of definition source architecture and target architecture. In this table, are presented the architecture requirements and your respective candidate solutions. All they save relation with the Archimate model presented.

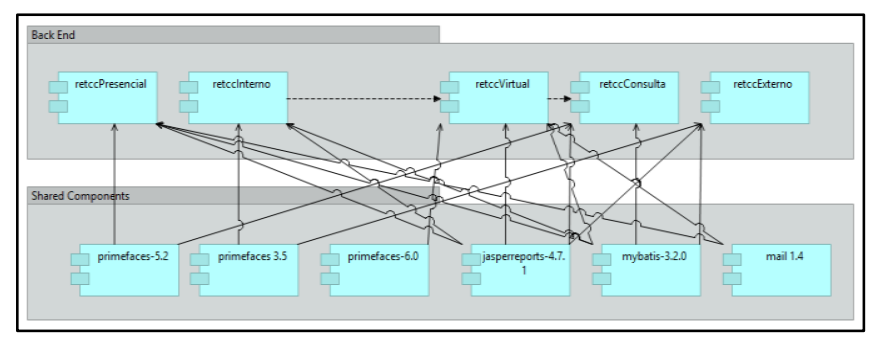

Fig. 5. Archimate AS IS Worker Recording Application Cooperation Viewpoint.

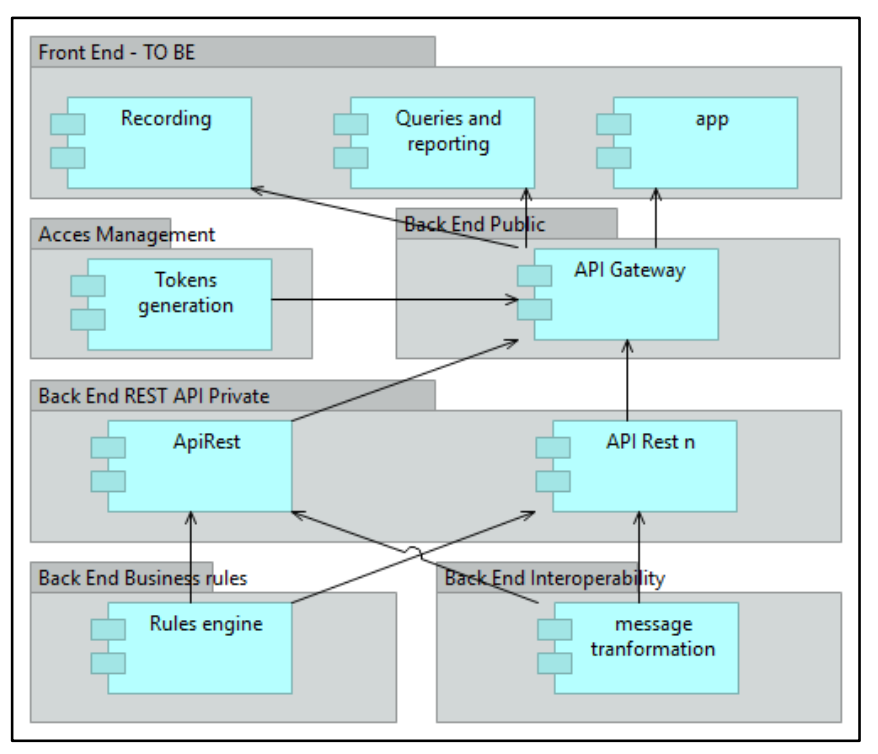

Fig. 6. Archimate TO BE Worker Recording Application Cooperation Viewpoint.

TABLE II. ARCHITECTURE GAP ANALYSIS

\begin{tabular}{|c|c|c|c|c|}
\hline & \multicolumn{3}{|c|}{ Target Architecture (TO BE) } \\
\hline & & $\begin{array}{l}\text { Worker Cross } \\
\text { Registration }\end{array}$ & $\begin{array}{l}\text { Rules Engine } \\
\text { Component }\end{array}$ & Removed \\
\hline \multirow{3}{*}{ 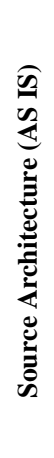 } & $\begin{array}{l}\text { C.C. Worker } \\
\text { Registration }\end{array}$ & Migrate & & $\begin{array}{l}\text { Remove in } \\
\text { medium } \\
\text { term }\end{array}$ \\
\hline & $\begin{array}{l}\text { CC Data } \\
\text { Calification }\end{array}$ & & Migrate & $\begin{array}{l}\text { Remove in } \\
\text { medium } \\
\text { term }\end{array}$ \\
\hline & New & $\begin{array}{l}\text { Transversal } \\
\text { capacity of } \\
\text { worker } \\
\text { registration }\end{array}$ & $\begin{array}{l}\text { Ability to } \\
\text { update and } \\
\text { reuse business } \\
\text { rules } \\
\text { throughout the } \\
\text { processes }\end{array}$ & \\
\hline
\end{tabular}

\section{Demonstration and Evaluation}

As a result of the model developed with Archimate realizing TOGAF ADM exercise, the usefulness of Archimate viewpoints and artifacts has been demonstrated to generate value for the aforementioned government agency, such as designing cross-sectional services for citizens. The evaluation of the proposal to confirm its added value is executed through the method used to map quality characteristics. Bunge-WandWeber method [19] is used to allows the mapping of following attributes of the Archimate model: Incompleteness, redundancy, excess and overload. Also, Moody and Shanks framework [20] is used to manage the quality of the model in: completeness, integrity, flexibility, understanding, correctness, simplicity, integration and implementability. The resulting evaluation is that the Archimate views presented are qualitatively satisfactory. 


\section{CONCLUSIONS AND FUTURE WORK}

With the joint application of TOGAF and Archimate, the objectives pursued by the solution proposal have been met: 1) Ensure alignment between strategic motivators and business processes and services, 2) Ensure alignment between processes and services of business with the applications and data, 3) Achieve and contribute to redesign citizen's cross-cutting services, standardize and make interoperable the corresponding information systems. The exposed knowledge can serve as a base to support new similar initiatives in the framework of better services to the citizen in the Peruvian state.

\section{ACKNOWLEDGMENT}

Thanks to the General Office of Statistics and Information Technology and Communications (OGETIC) of the Ministry of Labor and Employment Promotion of Peru for having an opening to develop a complete exercise of enterprise architecture development and as a result this article has been achieved.

\section{REFERENCES}

[1] OECD Public Governance Reviews, OECD Public Governance Reviews: Peru. Paris: OECD, 2016.

[2] US-GAO, "A Framework for Assessing and Improving Enterprise Architecture Management (Version 2.0)," United States Gov. Account. Off., no. August, p. 93, 2010.

[3] Diario El Peruano, "Decreto legislativo $\mathrm{N}^{\circ}$ 1412. Decreto legislativ que aprueba la Ley de Gobierno Digital,” El Peru., pp. 939-946, 2018.

[4] Y. Lee, Y. Kwon, S. Shin, and E. Kim, "Advancing Government-wide Enterprise Architecture - A Meta-model Approach,” 2005.

[5] K. Liimatainen, M. Hoffmann, and J. Heikkila, Overview of Enterprise Architecture Work in 15 Countries. 2007.

[6] Federal government of the United States, "Federal Enterprise Architecture Framework Version 2," Fed. Gov. United States, p. 434, 2013.
[7] A. Government and I. Management, "Australian Government Architecture Reference Models How to Use Guide," no. August, pp. 140, 2011.

[8] E. Architecture and U. K. G. Organisations, "Goals: Doing the Right Things Right," From Enterp. Archit. to IT Gov., pp. 43-73, 2007.

[9] M. F. Wong, "Enterprise Architecture Landscape in Singapore Government Agencies," 2013.

[10] G. of I. Ministry of Electronics and Information, "IndEA ... for ONE Government," Ministry of Electronics and Information Technology (MeitY), Government of India (GoI), 2018.

[11] D. C. L.M.Morales Moreno, J.O.Torres Páez, A.Parra, "The Colombian Government Enterprise Architecture Framework," 2014.

[12] T. Biggert, "Using Enterprise Architecture to Transform Service Delivery," Glob. Bus., pp. 311-343, 2011.

[13] K. Peffers, T. Tuunanen, M. A. Rothenberger, and S. Chatterjee, " $<$ Design Science Research Methodology 2008.pdf $>$," Publ. J. Manag. Inf. Syst., vol. 24, no. 3, pp. 45-78, 2007.

[14] D. H. Olsen, "Enterprise Architecture management challenges in the Norwegian health sector," Procedia Comput. Sci., vol. 121, pp. 637645, 2017.

[15] P. Saha, "Enterprise Architecture as a Platform for Connected Government: A Qualitative Analysis," p. 49, 2010.

[16] A. Ask and K. Hedström, "Taking initial steps towards enterprise architecture in local government," Lect. Notes Comput. Sci. (including Subser. Lect. Notes Artif. Intell. Lect. Notes Bioinformatics), vol. 6866 LNCS, pp. 26-40, 2011.

[17] A. K. S. Ajer and D. H. Olsen, "Enterprise Architecture Challenges: A Case Study of Three Norwegian Public Sector," Twenty-Sixth Eur. Conf. Inf. Syst., vol. 1, no. August 2018, 2018.

[18] S. R. Alan R. Hevner, Salvatore T. March, Jinsoo Park, "Design science in information systems research," MIS Q., 2004.

[19] Y. Wand and R. Weber, "On the ontological expressiveness of information systems analysis and design grammars," Inf. Syst. J., vol. 3, no. 4, pp. 217-237, Oct. 1993.

[20] D. L. Moody and G. G. Shanks, "Improving the quality of data models: empirical validation of a quality management framework," Inf. Syst., vol. 28 , no. 6, pp. 619-650, Sep. 2003. 